\title{
ABOUT THE ROLE OF THE SURFACE SCATTERING OF ELECTRONS ON THE EFFECT OF ELECTROMAGNETIC GENERATION OF SOUND
}

\author{
O. Galbova, G. Ivanovski \\ Institute of Physics, Faculty of Natural Sciences, P. O. Box 162 \\ Ss Cyril and Methodius University, Skopje, Macedonia
}

(Received April 3, 1998)

\begin{abstract}
A system consisting of sandwich structure: a halfspace (a dielectric or a conductor medium) and $N-1$ conductor films of thickness $d$ is considered. A recursive relation for the sound wave amplitude in the case of specular reflection of the conduction electrons is derived. The dependence of the amplitude of the sound field as a function of the number of conductor films is studied. A numerical analysis about the role of the surface scattering of electrons on the effect of electromagnetic generation of sound is performed.
\end{abstract}

Key words: electromagnetic wave, acoustic wave, diffuse scattering, sandwich structure.

PACS number(s): 72.10.-d, 72.50.+b

The elementary excitations in the conductors form a Fermi-liquid and their energy spectrum depends on the distribution function of the quasiparticles. Because of that, the response of the electron system to the external perturbation depends essentially on the correlation functions which describe the interaction between charge carriers [1, 2]. Usually, the Fermi-liquid interaction leads to renormalization of the kinetic coefficients which are derived assuming that conduction electrons form Fermigas. Nevertheless, in some cases, Fermi-liquid interaction leads to specific effects [3, 4].

From the electromechanical point of view, the conduction electrons and crystal lattice ions are in dynamical equilibrium. Namely, the lattice deformation leads to forces appearance which affect the conduction electrons. On the other side, the conduction electrons which are removed from equilibrium, affect the crystal lattice. As a result, in the equations of the theory of elasticity, terms which are functional of the distribution function of conduction electrons appear.

From the mathematical point of view, the connection of these subsystems is described by a system of equations formed by Maxwell equations, equations of the theory of elasticity, and the Boltzmann transport equation for conduction electrons [5,6].

One of the most expressive effects caused by interaction of these subsystems is the mutual transformation of electromagnetic and acoustic energy [7]. This effect is especially important as regards direct electromagnetic generation of high-frequency sound waves.

The investigations have been conducted in the absence of a constant magnetic field (when the contribution of the deformation mechanism is primary) as well as in the presence of a constant magnetic field (when the contribution of the induction mechanism is primary).

The surface defects are positions where the conduction electrons lose additionally their momentum gained from electromagnetic field. In the process of diffuse surface scattering of the conduction electrons, a $\delta$-shaped surface force appears [8]. This force contribution in the transformation of the electromagnetic energy into the acoustic energy is dominant in the case when $\omega \tau>1$ ( $\omega$ is the frequency of the electromagnetic field, $\tau$ is the relaxation time of electrons) [9, 10].

In different fields of investigation, the systems with sandwich structure: metal and dielectric or semiconductive layers, become more and more significant. On the other hand, in the last years, the interest from threedimensional structures has moved to the structures with smaller dimension [11].

It is obvious that analytical treatment of electroacoustic phenomena in sandwich structures becomes very difficult and for their investigating we have to use a computer technology. In the suggested study the first step in a long-term programme has been done. The aim of this work is to investigate the role of the surface scattering of conduction electrons in sandwich structures. For the sake of simplicity, we consider a model where each electron remains "captured" in its layer. In a more realistic model, which investigated now the conduction electrons should be able to tunnel through boundary surfaces with certain probability.

In the present work is considered a system consisting of a sandwich structure: half-space (dielectric or conductor) and $N-1$ conductor films of the thickness $d$, with the surface $[z=-(N-1) d]$ irradiated at an incident angle of $90^{\circ}$ by an electromagnetic wave with the frequency $\omega$. The $z$ - axis is taken to be parallel to the high-symmetry axis of the films and half-space medium, and accordingly the field in the sample has the polarization $\mathbf{E}_{m}=\left\{E_{m}(z), 0,0\right\}$. In order to find the displacement $u_{0}(z)$ in the half-space, we have to solve a set of equations formed by Boltzmann transport equation for conduction electrons written in $\tau$-approximation

$$
\frac{\partial \chi_{m}}{\partial z}-i \frac{\omega_{m}^{*}}{v_{z}} \chi_{m}=\frac{e v_{x}}{v_{z}} E_{m}, \quad \omega_{m}^{*}=\omega+i \nu_{m}
$$




$$
m=0,1,2, \ldots, N-1
$$

where $\nu_{m}$ is frequency of relaxation of the conduction electrons, $\chi_{m}$ is the non-equilibrium additive term to the equilibrium distribution function $f_{0}$ in the $m$ th sample, $\mathbf{v}=\left\{v_{x}, v_{y}, v_{z}\right\}$ and $e$ is the velocity and elementary charge of electrons; Maxwell equations

$$
\frac{\partial^{2} E_{m}}{\partial z^{2}}+i \omega \mu_{0} j_{m}=0, \quad j_{m}=e\left\langle v_{x} \chi_{m}\right\rangle
$$

and equations of the theory of elasticity

$$
\frac{\partial^{2} u_{m}}{\partial z^{2}}+q_{m}^{2} u_{m}=\frac{1}{\varrho_{m} s_{m}^{2}}\left\langle\Lambda_{x z}^{m}\left\{i \frac{\omega_{m}^{*}}{v_{z}} \chi_{m}+\frac{e v_{x}}{v_{z}} E_{m}\right\}\right\rangle
$$

Here $q_{m}=\omega / s_{m}$ is the wave number, $s_{m}$ - the velocity of the acoustic wave in the $m$ th sample, $\varrho_{m}$ - density of the metal, and $\Lambda_{x z}^{m}$ is deformation potential.

The functions $\chi_{m}, E_{m}$, and $u_{m}$ satisfy the following boundary conditions:

1) In order to simplify the problem we consider the case when the electrons from the two contact-media are scattered without penetration through the contactsurfaces. So, in the framework of the Fucks model [10], the boundary conditions for the non-equilibrium additive term to the equilibrium distribution function of conduction electrons are written in the form

$$
\begin{gathered}
\chi_{m}\left(\mathbf{r}_{s}, \mathbf{p}^{-}\right)=\mathcal{P} \chi_{m}\left(\mathbf{r}_{s}, \mathbf{p}^{+}\right), \\
m=0,1,2, \ldots, N-1,
\end{gathered}
$$

where $\mathbf{r}_{s}$ is the radius vector of electrons on the boundary surfaces, and $\mathcal{P}$ is a coefficient which describes the ability of the scattering on the surfaces $(0 \leq \mathcal{P} \leq 1)$.

2) The equality of tangent component of the electric $E_{m}(z)$ and magnetic $H_{m}(z)$ components of the electromagnetic field, i.e.

$$
\begin{gathered}
E_{m}(-m d)=E_{m+1}(-m d), \\
H_{m}(-m d)=H_{m+1}(-m d), \\
(m=0,1,2, \ldots, N-2),
\end{gathered}
$$

where $\mathbf{H}_{m}=\left\{0, H_{m}, 0\right\}$ is a component of the magnetic field in the $m$ th sample.

3) The boundary conditions for sound displacement

$$
u_{m}(-m d)=u_{m+1}(-m d),
$$

$$
\begin{gathered}
\left.\varrho_{\mathrm{N}-1} s_{\mathrm{N}-1}^{2} \frac{d u_{\mathrm{N}-1}(z)}{d z}\right|_{z=-(N-1) d} \\
=\frac{\mathcal{P}-1}{\mathcal{P}+1}\left\langle\left.\frac{\Lambda_{x z}^{(N-1)} v_{z}}{\left|v_{z}\right|} \chi_{\mathrm{N}-1}(z)\right|_{z=-(N-1) d}\right\rangle, \\
\varrho_{m} s_{m}^{2} \frac{d u_{m}(-m d)}{d z}-\frac{\mathcal{P}-1}{\mathcal{P}+1}\left\langle\frac{\Lambda_{x z}^{(m)} v_{z}}{\left|v_{z}\right|} \chi_{m}(-m d)\right\rangle \\
=\varrho_{m+1} s_{m+1}^{2} \frac{d u_{m+1}(-m d)}{d z} \\
\quad-\frac{\mathcal{P}-1}{\mathcal{P}+1}\left\langle\frac{\Lambda_{x z}^{(m+1)} v_{z}}{\left|v_{z}\right|} \chi_{m+1}(-m d)\right\rangle, \\
m=0,1,2, \ldots, N-2 .
\end{gathered}
$$

To solve the system of equations (1)-(3) which satisfies the boundary conditions (4)-(6) the Fourier method is used.

With the successive solutions of the problem, for $1,2,3, \ldots, N-1$ conductive films spread over a metal half-space, a recursive relation for the sound wave amplitude in the case of specular surface reflection of the conduction electrons is derived. A "Mathematica"-coded computer program is written and used in the calculation of the sound field amplitude. By means of numerical methods, the expression for an arbitrary number of isotropic conductor layers is analysed in the whole region of the relevant parameters determining the magnitude of the electromagnetic generation: the depth of the film $d$, the working temperature and the frequency of the electromagnetic wave. The numerical results obtained for metal film and metal half-space are in good agreement with some previously obtained results [8].

In the process of diffuse surface scattering of the conduction electrons, a $\delta$-shaped surface force could appear. The effect of electromagnetic generation of sound is governed by the forces appearing in the vicinity of the sample surface, at which the conduction electrons are scattered. This situation appears not only at the samplevacuum boundary, but at any boundary that may be treated as a surface defect. Therefore, it is of interest in considered sandwich structure, to introduce a certain "diffusity" in the scattering of conductor electrons.

An analytical relation for the sound wave amplitude in the systems with $N=2$ and $N=3$ isotropic conductor layers for non-specular surface scattering of the conduction electrons is written. They will not be presented here because of their quantity. We will analyze these results numerically in a text below.

"Mathematica"-coded computer programs are also written for calculation of the sound field amplitude in the systems with $N=2$ and $N=3$ layers. These programs allow to analyze the acoustic field amplitude without limits of the relevant parameters which describe electromagnetic generation of sound. 
The numerical analysis shows that for frequencies of electromagnetic field $\omega$ smaller than relaxation frequency $\nu(\omega<\nu)$ and the diffuse scattering of the electrons have no influence over the result derived for pure specular reflection. The role of the surface scattering of electrons on the effect of electromagnetic generation of sound is important when $\omega>\nu$.

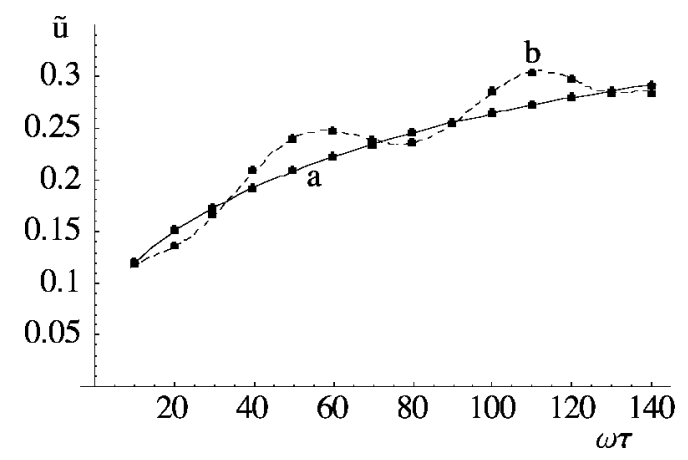

Fig. 1. The frequency dependence of the sound wave amplitude $(N=2, d<\delta<\lambda<\ell)$.

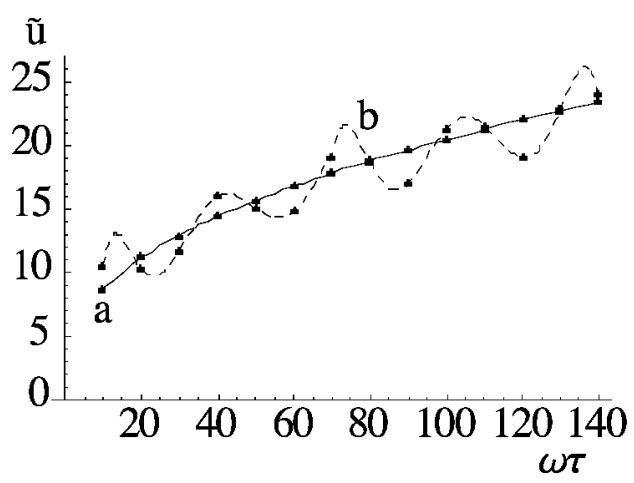

Fig. 2. The frequency dependence of the sound wave amplitude $(N=2, d<\delta<\ell<\lambda)$.

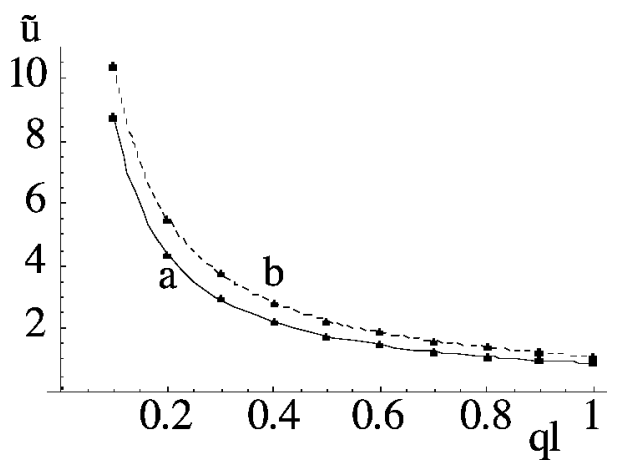

Fig. 3. The amplitude dependence of $\ell(N=2$, $d<\delta<\ell<\lambda$ ).

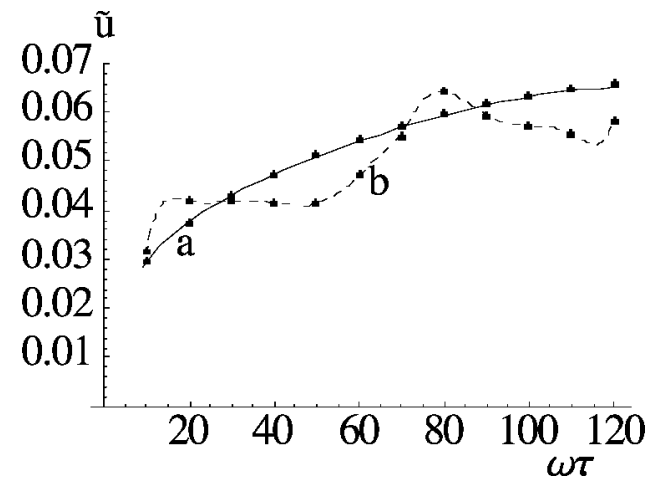

Fig. 4. The frequency dependence of the sound wave amplitude $(N=3, d<\delta<\lambda<\ell)$.

Fig. 1 and 2 give the frequency dependence of the sound amplitude $\widetilde{u}$ ( $\widetilde{u}$ is renormalized sound amplitude) for different value of parameters in a system with $N=2$. The first one is related to the case when the highest parameter with the dimension of length is mean free path of conduction electrons $\ell(d<\delta<\lambda<\ell, \delta$ is skin depth), and the second one - when the highest parameter with the dimension of length is the sound wave length $\lambda(d<\delta<\ell<\lambda)$. The curves $a$ give the frequency dependence of the field for pure specular reflection $(\mathcal{P}=1)$, and the curves $b$ - for non-specular reflection $(\mathcal{P}=0.8)$. The graphs show that the amplitude of the sound field, for non-specular reflection, is an oscillatory function of the frequency. At certain frequencies, the coupled electromechanical forces (arising from the diffuse scattering of the electrons) may result in amplified or decayed sound generation.

Fig. 3 gives the amplitude dependence on free mean path $\ell$ for the same cases. A graph shows that the diffuse scattering does not change the amplitude dependence of $\ell$, but it has significant influence on the value of sound wave amplitude.

Fig. 4 gives the frequency dependence of the sound amplitude $\bar{u}$ in a system consisting of two conductor films $(N=3)$. The curve a gives the frequency dependence of the field for pure specular reflection $(\mathcal{P}=1)$, and the curve $b-$ for non-specular reflection $(\mathcal{P}=0.8)$. A graph shows that the height of the maxima compared with the monotonous part is more pronounced in the case of two films. We can also see that the increase in the number of conductor films, leads to a decrease of the sound field amplitude. This result is a consequence of the employed model (the electrons are scattered without penetration through the contact-surfaces). Further improvements of the model are desirable introducing, in the limiting conditions, certain probability for tunnelling of the conduction electrons between the layers.

This research was carried out under financing by the Ministry of Sciences of Macedonia. 
[1] L. D. Landau, Zh. Eksp. Teor. Fiz. 30, 10 (1956).

[2] V. P. Silin, Zh. Eksp. Teor. Fiz. 38, 977 (1960).

[3] V. P. Silin, Usp. Fiz. Nauk 93, 185 (1967).

[4] V. N. Bagaev, V. I. Okulov, E. A. Pamyatnih, Pis'ma Zh. Eksp. Teor. Fiz. 27, 156 (1978).

[5] V. M. Kontorovich, Usp. Fiz. Nauk 142, 266 (1984).

[6] A. F. Andreev, D. I. Pushkarov, Zh. Eksp. Teor. Fiz. 89, 1883 (1985).

[7] A. N. Vasil'ev, Yu. P. Gaidukov, Usp. Fiz. Nauk 141,
431 (1983).

[8] G. Ivanovski, M. I. Kaganov, V. B. Fiks, Fiz. Tverd. Tela 15, 1441 (1973).

[9] G. Ivanovski, M. I. Kaganov, Fiz. Tverd. Tela 18, 2704 (1976).

[10] G. Ivanovski, O. Galbova, Phys. Stat. Solidi B 134, 815 (1986).

[11] V. M. Gokhfeld, O. V. Kirichenko and V. G. Peschansky, Zh. Eksp. Teor. Fiz. 108, 2147 (1995). 